\title{
Comparison of time-lapse GPR and resistivity over simulated clandestine graves
}

Pringle, J.K. ${ }^{1}$, Jervis, J.R. ${ }^{1}$ \& Tuckwell, G.T. ${ }^{2}$

${ }^{1}$ School of Physical Sciences \& Geography, Keele University, Keele, Staffs., ST5 5BG, UK.

${ }^{2}$ Stats Limited, Porterswood House, St. Albans, Hertfordshire AL3 6PO, U.K.

Email: j.k.pringle@esci.keele.ac.uk,j.jervis@epsam.keele.ac.uk

\& george.tuckwell@stats.co.uk

\section{Summary}

Forensic geophysics should be an invaluable tool to assist search teams to detect and locate clandestine graves of buried murder victims. At present however, geophysics is underutilised and currently used techniques may not be optimal for specific targets or sites. There is a need for geophysical datasets to be collected over known burial sites for varying time periods post-burial.

A study site was created with a naked and wrapped pig cadaver. The dimensions are based on available statistics of discovered burials. Monthly surveys using resistivity, Electrical Resistivity Tomography (ERT) and Ground Penetrating Radar (GPR) were performed postburial. Resistivity results show low anomalies over the naked pig and a smaller high anomaly over the wrapped pig with respect to background values. ERT time-lapse data shows optimum survey periods for the naked and wrapped pigs to be 9 and 3 months respectively. GPR 2D profiles detected both burials, with the wrapped pig exhibiting stronger reflection events. Lower frequency $(110 \mathrm{MHz})$ antennae were found to be the optimal frequency to detect pig burials.

\section{Main Objectives:}

1. Detail time-lapse geophysical results over simulated clandestine graves.

2. Determine optimal method(s) to locate a grave post-burial and if this changes over time.

3. Determine which frequency GPR antennae is optimal.

4. Compare GPR with ERT surveys.

\section{New Aspects Covered:}

Creating base-line geophysical datasets in this rigorous fashion (monthly) and sitespecific factors (local climate, moisture content, porosity) etc will allow results to be applied to real case studies. Other researchers rarely combine resistivity (lateral resistivity \& ERT) and GPR repeat surveys. Comparing wrapped \& naked cadavers has also not be previously investigated \& published.

\section{Topics}

1. Archeo and Forensic Geophysics

2. Electrical, Electromagnetic and GPR Studies 


\section{Introduction}

Forensic geophysical investigations should be an invaluable tool to assist search teams to detect and locate clandestine graves of buried murder victims. At present however, geophysics is under-utilised. Additionally, common techniques may not be optimal for specific targets or sites. This is particularly relevant for UK soil types that have high clay contents which attenuate Ground Penetrating Radar (GPR) signals or urban sites with significant near-surface objects (Pringle et al., 2008). GPR is typically the most popular for clandestine grave detection, but electrical methods are a useful alternative. There is a need for forensic geophysical datasets to be collected over known burial site(s) and for the collection of geophysical data over varying time periods post-burial. This would assist forensic practitioners to decide which technique to use for specific sites, based on the geophysical response of the known graves and how this changes over time.

Geophysical surveys over simulated clandestine graves provide useful results, as the target size, age and location are known. In the US, human cadavers have been used in studies within mostly sandy soils (e.g France et al., 1992). Due to the Human Tissue Act (2005) precluding the use of human remains in the UK, human proxies are replaced with domestic pig cadaveres. Lateral resistivity mapping results are shown elsewhere (Jervis et al., in review). This paper reports on the GPR and Electrical Resistivity Tomography (ERT) baseline surveys over simulated burial sites over a year. A secondary aim is to compare different GPR frequency datasets and to determine which frequency is optimal.

\section{Method}

The study was undertaken within a controlled test site on Keele University campus in the UK (Figure 1). The site is underlain by the Triassic Keele Sandstone Formation which lies $\sim 4 \mathrm{~m}$ below ground level (bgl), based on nearby borehole records. The overlying, predominantly sandy loam soil is partly made ground, due to demolished greenhouse shallow foundations.

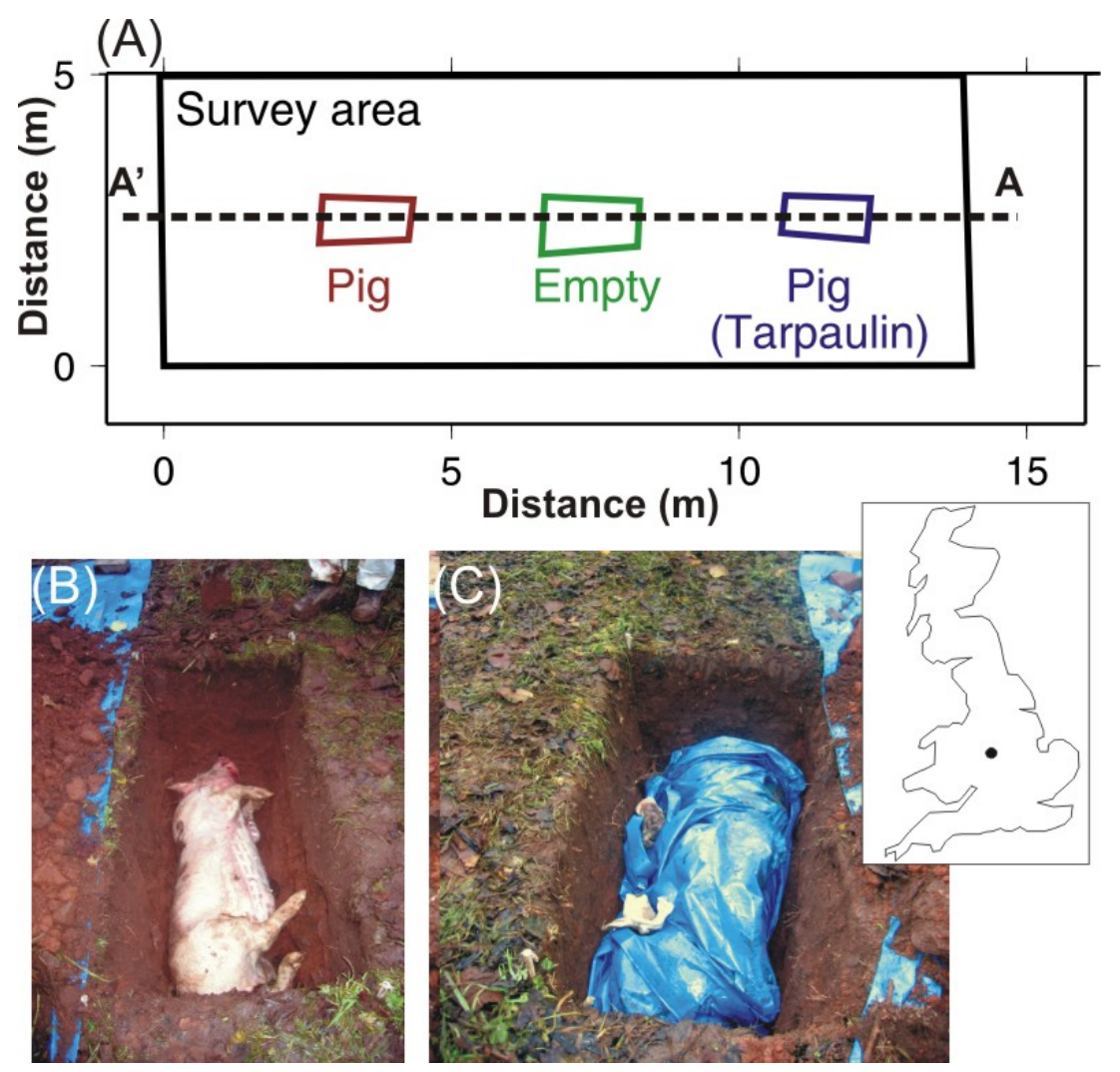

Figure 1 (A) Study site map (location map inset) with (B) naked and (C) wrapped pig grave photographs respectively. 
The survey area contained three graves (Figure 1), one being empty to determine whether the geophysical results were influence by disturbed ground. The other two graves contained $80 \mathrm{~kg}$ pig cadavers; one naked and one wrapped in a tarpaulin sheet. One cadaver was wrapped because available statistics of discovered burials show $50 \%$ are either wrapped or clothed (Manhein, 1996). Depth of burial was $0.5 \mathrm{~m} \mathrm{bgl}$, again based on average discovered depths of available statistics (Komar, 2003). The nearby Keele meteorological weather station supplied total rainfall and $0.5 \mathrm{~m}$ bgl temperature information over the study period (Figure 2).

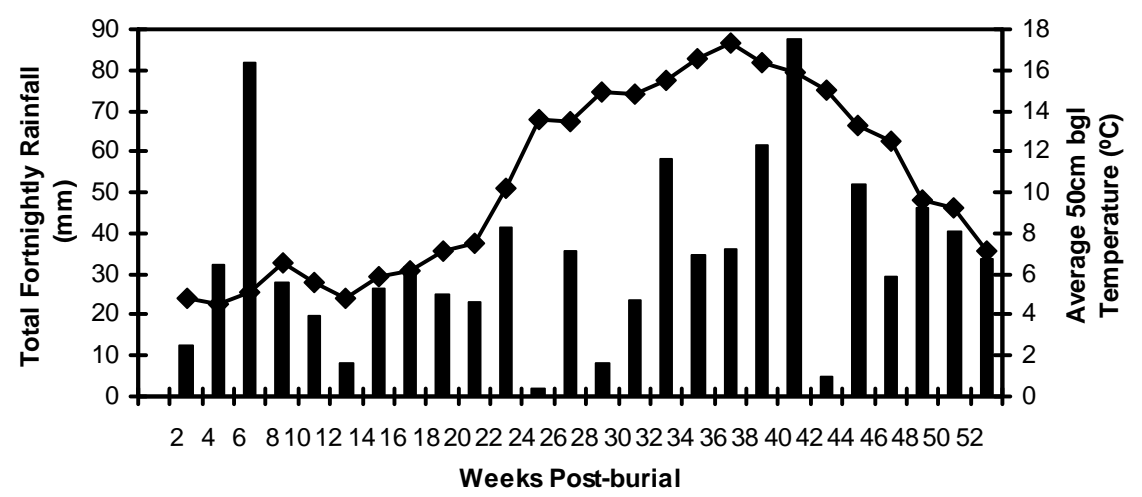

Figure 2 Summary of weather data over the study period.

Bulk ground resistivity and GPR surveys were conducted prior to burial (to act as control) and at monthly intervals post-burial. Every three months 2D Electrical Resistivity Tomography (ERT) profiles were collected. Campus TIGRETM ERT arrays had a $0.5 \mathrm{~m}$ electrode separation and 10 depth levels. GPR surveys used PulseEKKO ${ }^{\mathrm{TM}} 1000$ equipment and 110, 225, 450 and $900 \mathrm{MHz}$ dominant frequency data were collected separately. Every 0.5m 2D GPR profiles were collected, with trace spacings for the different frequencies at $0.20 \mathrm{~m}, 0.1 \mathrm{~m}$, $0.05 \mathrm{~m}$ and $0.025 \mathrm{~m}$ respectively. Standard GPR processing steps were undertaken (Pringle et al., 2008), as well as more advanced steps of migration and time-slice generation.

\section{Results}

Selections of processed but un-migrated 2D GPR profiles are shown in Figure 3. Clearly obvious parabolic reflection events are obtained from all frequencies over the burials. The lower frequency datasets show the targets but very few other features. As a result, the migrated $110 \mathrm{MHz}$ time slices show the graves clearer than higher frequencies (Figure 4).

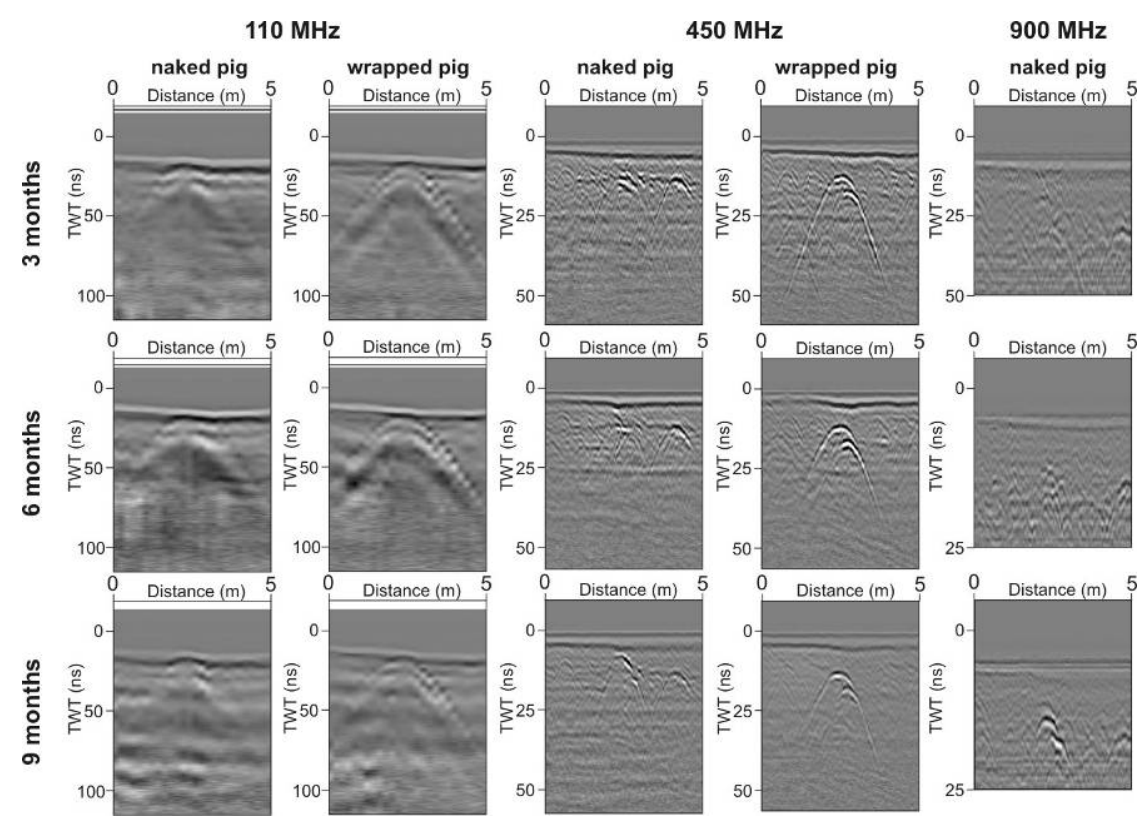

Figure 3 Selected GPR 2D Profiles 


\section{Near Surface}

$110 \mathrm{MHz}$
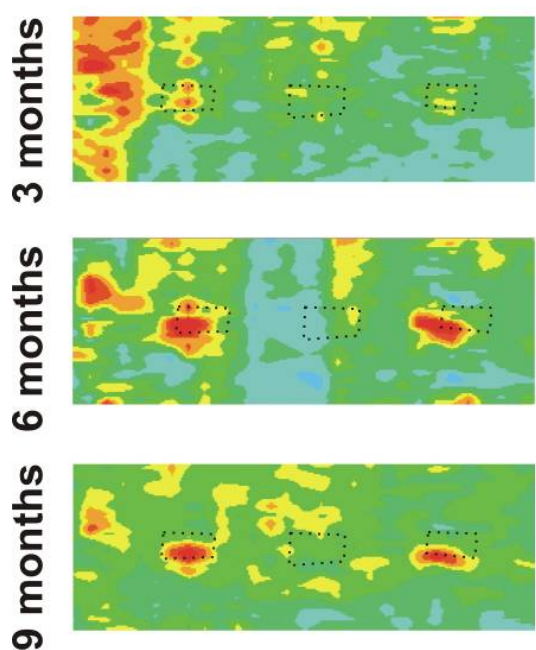

$450 \mathrm{MHz}$
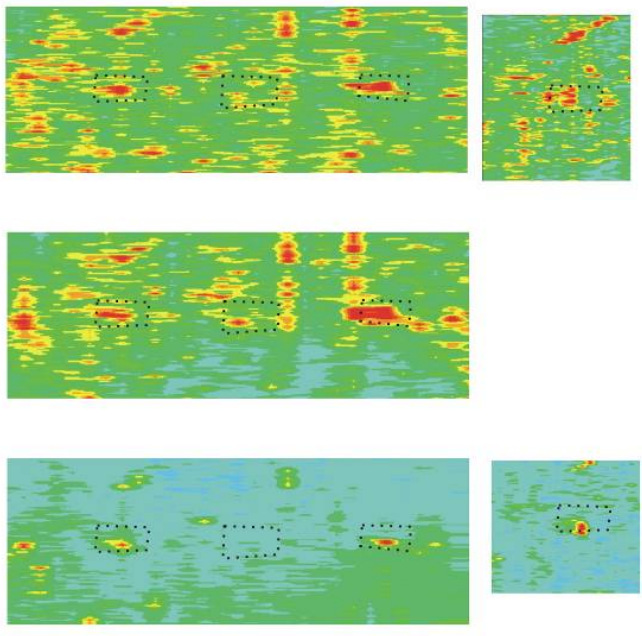

Figure 4 Selected GPR time-slices (grave positions marked)
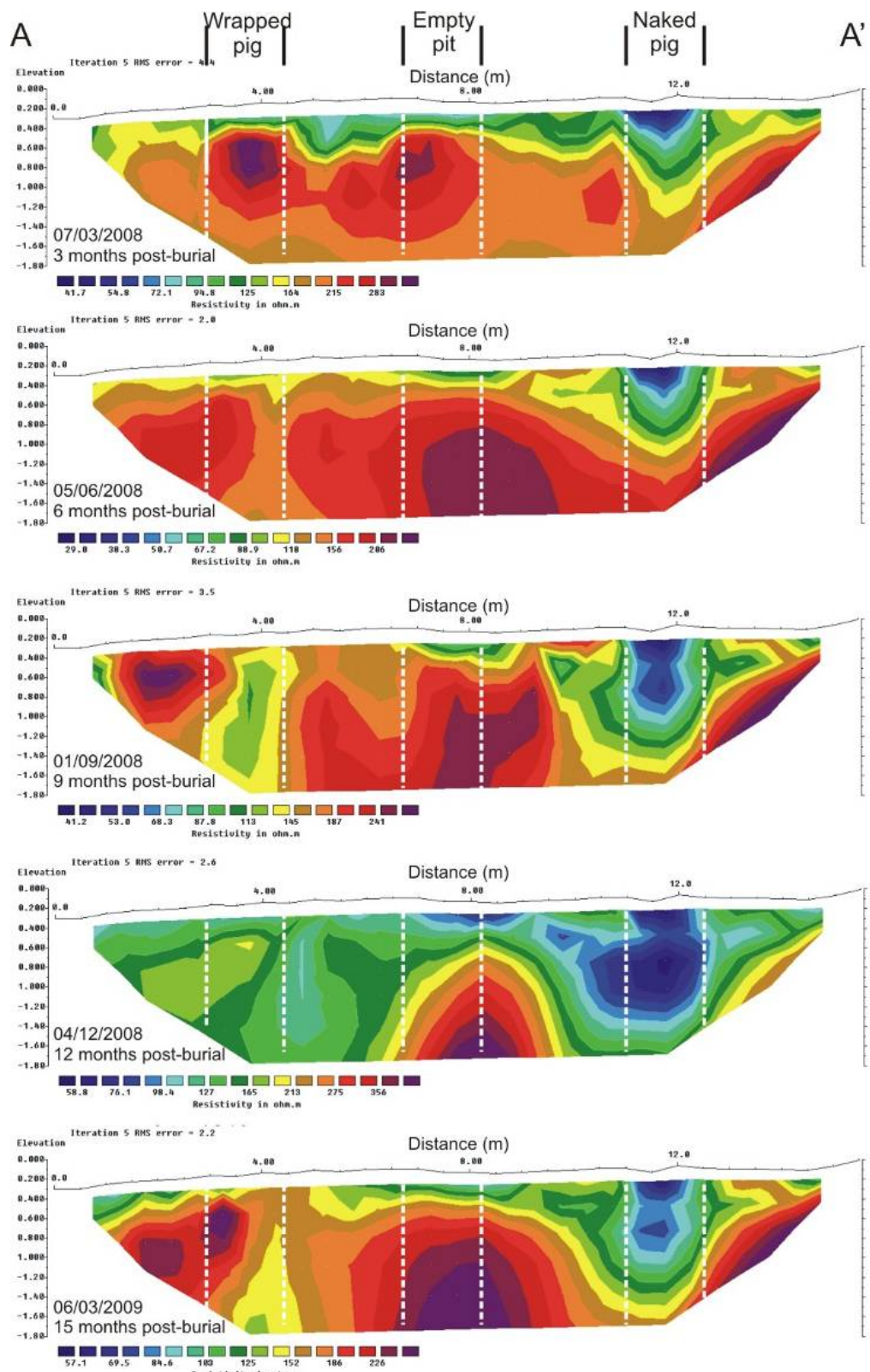

Figure 5 Time-lapse ERT profiles (see location map). 
Electrical resistivity results shown elsewhere (Jervis et al., in review) observe low resistivity anomalies over the naked pig and high anomalies over the wrapped pig with respect to background values. This is also observed but in more detail with the ERT results (Figure 5). The lowest resistivity low anomaly over the naked pig is observed nine months post-burial. The strongest high anomaly over the wrapped pig is observed three months post-burial. The wrapped pig anomaly is also smaller and less strong than the naked pig anomaly.

\section{Forensic search implications}

Forensic GPR studies commonly use medium to high (225-900 MHz) frequencies. We suggest using lower frequency antennae (110 MHz in this case) if the target size is significant. Not only are low frequency GPR data quicker to acquire, saving time and resources, but there is significantly less clutter in the data. There are differences detected from GPR responses over the wrapped and naked pig graves, both in signal amplitude and reflection event consistency. This is most probably due to the wrapping material providing a stronger contrast than the naked decomposing cadaver. The observed strong time-slice signal amplitudes over the pig graves from the six to nine month post-burial datasets also indicate optimal periods to survey a target post-suspected burial.

The constant separation and ERT resistivity surveys detected both the naked and wrapped pig graves, although the wrapped pig was harder to detect. Resistivity can be a viable alternative to GPR surveys, particularly if the soil clay content is high or there are significant other nontarget objects within the near-surface. ERT surveys give comparable resolutions to GPR data, with the strongest anomalies observed over the naked pig nine months post-burial. This will be associated with the increasingly conductive decomposition fluids (see Jervis et al., in review).

\section{Acknowledgements}

John Jervis' PhD is jointly funded by the EPSRC and Stats Limited. A HEFCE SRIF2 equipment grant funded purchase of the ERT equipment. The authors thank Tim Millington and Malcolm Wright for assistance in creating the study site.

\section{References}

France, D.L., Griffin, T.J., Swanburg, J.G., Lindemann, J.W., et al., [1992] A multidisciplinary approach to the detection of clandestine graves. Journal of Forensic Sciences, 37, 6, 1445-58.

Jervis, J.R., Pringle, J.K. \& Tuckwell, G.W. [In review] Time-lapse resistivity surveys over simulated clandestine graves. Forensic Science International.

Komar, D.A. [2003] Twenty-seven years of forensic anthropology casework in New Mexico. Journal of Forensic Science, 48, 3, 521-524.

Manhein, M.H. [1996] Decomposition rates of deliberate burials: a case study of preservation. In: Haglund WD \& Sorg MH (Eds) Forensic taphonomy: the post-mortem fate of human remains. Boca Raton, FL: CRC Press LLC, 469-81.

Pringle, J.K., Jervis, J., Cassella, J.P. \& Cassidy, N.P. [2008] Time-lapse geophysical investigations over a simulated urban clandestine grave. Journal of Forensic Science, 53, 6, 1405-12. 